\title{
Preface
}

\section{Basics of Alzheimer's Disease Prevention}

In this special issue of the Journal of Alzheimer's Disease, a panel of international experts was convened to review the major pillars of prevention of Alzheimer's disease (AD). These pillars, and their corresponding sections, are as follows:

1. Identification and relative impact of AD risk factors;

2. Pathology associated with AD risk factors;

3. Detection of AD risk factors;

4. Interventions to delay or prevent risk factor progress.

Despite the glacial pace of clinical progress over the last 100 years, it is encouraging that our present knowledge of AD risk factors offers fertile avenues of exploration that can focus on significant interventions to help delay or prevent the onset of dementia.

Modifiable risk factors include type 2 diabetes, atherosclerosis, hyperlipidemia, hypertension, hypercholesterolemia, cardiac disorders, cerebrovascular pathology, body mass index, and an assortment of unhealthy lifestyle conditions. However, mere knowledge of $\mathrm{AD}$ risk factors is prognostically fruitless if their detection cannot be made using sensitive and costeffective markers for mass screening. Ideally, such risk factor detection should focus on asymptomatic or mildly cognitive dysfunctional individuals where the chance of successful intervention is greater. Just as the harmful consequences of cervical cancer, pancreatitis, severe anemia, and acute renal failure can be generally improved through routine lab test detection and administration of appropriate therapy, so too can preclinical AD become responsive to early detection of risks factors and targeted treatments aimed at reducing the severity and progress of the discovered pathology. Unlike the potentially lethal disorders stated above, people with AD have no treatment options-prevention thus becomes an alternate and essential primary weapon to combat this disease.
Two pitfalls associated with AD prevention should be avoided. First, the idea often presented in the literature that a universal tonic can be formulated to prevent sporadic $\mathrm{AD}$ in all individuals fails to recognize that this dementia is multifactorial with poly-etiological risk factors that contribute to the cause of this dementia. Second, the idea promoted by some enterprising pharmaceuticals of finding a "cure" for AD fails to grasp or simply ignores the reality that dead neuronal networks responsible for the advanced cognitive loss cannot be replaced without altering the intellectual capacities and personal identity of the affected individual. This is true even if neurogenesis, stem cell, or any other neuroregenerative scheme replaces the fatally damaged AD neurons.

When prevention or delay of cognitive deterioration is not possible for any reason, it is the duty of the practitioner to try to provide any therapeutic relief that "works" to ease the suffering caused by dementia. This may be difficult because the bottom-line message that emerges from all AD research done so far is that the status quo has brought virtually nothing worthwhile to the clinical outlook of AD. This bleakness of prognosis is partly promoted by the monetary magnet to market a blockbuster 'cure' for $\mathrm{AD}$, often at the expense of tortured data reminiscent of pounding a square peg in a round hole.

The knee-jerk dogma of the late $20^{t h}$ century, that $\mathrm{AD}$ is caused by amyloid toxicity, deserves to be treated with more skepticism in this century. So dominant in research has the amyloid hypothesis become that challengers of its tenet invite peer disdain or, like the fabled king in The Emperors' New Clothes, suspicion of consummate stupidity.

When clinical dogma limits enquiry and fosters circular reasoning, patients will suffer. This point is painfully clear when it is noted that billions of dollars have been spent by funding agencies to promote many dubious projects in dementia research. The consequence is that virtually nothing helpful has trickled 
down to the individual facing a diagnosis of AD. If any headway is to be made from bench-to-bedside research, funding agencies need to escape from the mentality of past failures. Common sense dictates that funding support should emphasize pragmatic solutions that clinicians can apply to patients at risk of dementia. Basic research is essential to open translational lines of opportunities that can help patients, but a fair balance between bench and bedside priorities is needed.

An initial and important step is taken in this special issue by its presentation of a plan to action to address the expanding prevalence of AD. The strategies proposed here are not silver bullets, but they offer a realistic hope to the millions of people who face a gauntlet of noxious maladies during aging - including multiple risk factors that can culminate in dementia.

The approach of knowledge-to-action using evidence-based medical decisions to opt for the most judicious treatments or management of patients when risk factors to $\mathrm{AD}$ are discovered, as reviewed in this issue, should become a gold standard of clinical practice. The integration of meta-analysis for interpretation of research data and decision-making and of Bayesian analysis for estimating future probability of acquiring adverse clinical conditions based on observed data, are tools that can further discourage the use of harmful or worthless treatments.

The success of early detection of risk factors and early treatment can improve when traditional meta- analysis or a Bayesian approach (when sparse data is available) is employed. This paradigm shift in clinical thinking substantially upgrades the level of preventive measures that can significantly delay cognitive decline.

In summary, an $\mathrm{AD}$ preventive plan would incorporate:

1. early identification/detection of AD risk factors;

2. early intervention based on evidence-based medical decisions;

3. patient follow-up to assess and modify when necessary, strategic intervention.

A qualified way to put preventive strategies on a practical scale is to develop a computer program using knowledge-to-action parameters where a patient's medical history and detected risk factor data are fed into the program. A best-fit recommendation of either tailoredmedical therapy or management would then be available to the practitioner for consideration. The design of such a program would remove much guesswork from clinical decisions of a generally complex nature and could provide a major opportunity to vastly slow down the avalanche of new AD cases.

Jack C. de la Torre, MD, PhD

Center for Alzheimer's Research

Banner Sun Health Research Institute

Sun City, AZ 85351, USA

jcdelatorre@comcast.net 